\title{
PREFERE-Studie: Was ist das Beste für den Patienten?
}

W

as ist im frühen Stadium des Prostatakarzinoms die beste Behandlungsoption? $\mathrm{Zu}$ dieser Frage gibt es derzeit keine klare Antwort, denn laut der aktuellen S3-Leitlinie für das lokal begrenzte Prostatakarzinom kommen vier Behandlungsmethoden in Betracht: die radikale Prostatektomie, die perkutane Strahlentherapie, die Bestrahlung von innen mittels implantierter "Seeds“ („low-dose-rate“-Brachytherapie) und Active Surveillance. Welche Methode wann angewendet wird, wird im Einzelfall entschieden. Eine evidenzbasierte Datenlage gibt es dazu nicht. Daher wird nun ein einzigartiges Projekt angescho- ben: Mithilfe einer bundesweiten Studie mit über 7.000 Teilnehmern und einem Beobachtungszeitraum von zehn Jahren soll erstmals nach den Maßstäben der evidenzbasierten Medizin verglichen werden, ob eine der vier geeigneten Alternativen bei der Behandlung des lokal begrenzten Prostatakrebses den anderen über- oder unterlegen ist. Der Studienbeginn der „präferenzbasierten randomisierten Studie beim NiedrigrisikoProstatakarzinom" (PREFERE) ist für Anfang 2012 vorgesehen. Studienleiter Prof. Michael Stöckle, Homburg, betonte: „Da man in der Niedrigrisiko-Gruppe sicherlich die höchste Rate an Überthera- pie zu erwarten hat, sind wir es unseren heutigen und unseren zukünftigen Prostatakrebs-Patienten schuldig, jede Anstrengung zu unternehmen, offene Fragen auf evidenzbasierter Grundlage zu klären." Eine Besonderheit ist die industrieunabhängige Finanzierung der Studie.

In Hamburg prognostizierte man, dass die Ergebnisse dieser Untersuchung die Behandlung des niedriggradigen Prostatakarzinoms auch international beeinflussen werden.

Bettina Reich

F 8: „Niedrigrisiko-Prostatakarzinom”

\section{Botulinumtoxin-Präparate bei OAB gleichwertig}

Dotulinumtoxin-A-Detrusorinjektionen sind eine Option in der Behandlung der therapierefraktären überaktiven Blase („overactive bladder“, OAB) und bislang zur Behandlung der neurogenen Blase bei Querschnittverletzten und MSPatienten zugelassen. Falls bei Patienten eine Tachyphylaxie auftritt, wie dies bei neurologischen Langzeitpatienten beobachtet wurde, könnte ein Produktwechsel die Unwirksamkeit des Botulinumtoxins theoretisch verzögern. Hierbei ist es wichtig, dass die verschiedenen Präparate ein vergleichbares Wirkungs- und Nebenwirkungsprofil haben.
PD Dr. med. Christian Hampel, Mainz, stellte eine Studie vor, in der die Wirkung von 300 U Onabotulinumtoxin A (Botox ${ }^{\otimes}$ ) und $300 \mathrm{U}$ Incobotulinumtoxin A (Xeo$\min ^{\circledast}$ ) bei 78 neurogenen und 92 nicht neurogenen Patienten mit urodynamisch verifizierter Detrusorinstabiliät verglichen wurde. Sechs Wochen nach dem Eingriff nahm die Miktionsfrequenz (Miktionen/24 h) in beiden Gruppen signifikant ab. Jedoch betrug die subjektive Patientenzufriedenheit in der Onabotulinumtoxin-A-Gruppe nur 47,4\%, im Vergleich zu $71,8 \%$ in der Incobotulinumtoxin-AGruppe. Hampel führte diese Diskrepanz unter anderem auf eine negative Patientenselektion zurück, da es zu Studienbeginn nur ein Präparat gab. Mit Zulassung von Xeomin ${ }^{\circledR}$ und erfolgter Randomisierung stieg die Zufriedenheit mit den Jahren an. Beide Präparate seien daher bei gleicher Dosis äquieffektiv. PD Dr. Jasmin Badawi, Luzern, wies darauf hin, dass auch theoretisch nicht mit einem Wirkunterschied zu rechnen sei, da Onabotulinumtoxin A nach der Injektion in das aktive $150-\mathrm{KDa}$ Neurotoxin zerfällt. Sebastian Lux

V 10: "Unterer Harntrakt, Urodynamik, Neurourologie - Therapie"

\section{S3-Leitlinie Prostatakarzinom: Neufassung in der Diskussion}

$\mathrm{D}$ ie schnelle Entwicklung von diagnostischen Verfahren und Therapieoptionen machen eine ständige Aktualisierung unserer Leitlinien notwendig", berichtete Prof. Manfred Wirth, Dresden. So wurden neue diagnostische Verfahren wie die Elastografie, das Histoscanning, die Magnetresonanztomografie (MRT), alternative Verfahren wie die Kryotherapie und hochintensiv-fokussierter Ultraschall (HIFU) neu bewertet.

Als neue Empfehlung wurde unter anderem dafür gestimmt, dass die Elasto- grafie, aber auch die Positronenemissionstomografie-Computertomografie (PET/CT) sowie die MRT noch nicht zur Primärdiagnostik eingesetzt werden sollte. Eine weitere neue Empfehlung besagt, dass der HIFU zur Therapie des histologisch gesicherten isolierten Lokalrezidivs nach perkutaner Strahlentherapie eingesetzt werden kann. Allerdings sollte der Patient über den experimentellen Charakter dieses Verfahrens als SalvageTherapie und über die Therapiealternativen informiert werden.
$\mathrm{Ab}$ sofort ist die Konsultationsfassung online auf den Internetseiten der DGU (www.dgu.de), der beteiligten Fachgesellschaften sowie des Ärztlichen Zentrums für Qualität in der Medizin (ÄZQ) einzusehen. Stellungnahmen und Änderungsvorschläge dazu werden berücksichtigt, sofern sie begründet und mit Literatur hinterlegt sind.

Bettina Reich

V 24: „Prostatakarzinom - Therapie fortgeschrittener Tumoren" 